\title{
Spermatic Cord Cyst Presenting as an Incarcerated Inguinal Hernia: An Unusual Case and Literature Review
}

\author{
Shane P Smith*, Katherine A Mandell and Michael G Florence \\ Swedish Medical Center, USA \\ *Corresponding author: Shane P Smith, Swedish Medical Center, 747 Broadway, 10 Heath, Seattle, WA 98122, USA, Tel: 206-518-3050; \\ Email: shane.smith@swedish.org
}

Submission: 阱 March 09, 2018; Published: 眥 March 16, 2018

\begin{abstract}
Inguinal cord cysts are a rare finding, comprising less than $1 \%$ of inguinal hernia dissections. These cysts are anatomically unique and distinct from cord lipomas and hydroceles. They are most commonly benign masses that contain atypical tissue and clinically present as incarcerated hernias. A patient was taken to surgery for repair of suspected incarcerated inguinal hernia and found to have no hernia but a cystic mass on the spermatic cord. Final histopathological diagnosis was pseudocyst. The patient recovered well with resolution of pain confirmed at his postoperative follow up. An inguinal cord cyst is a rare and surprising finding during routine inguinal hernia surgery. The pain and inability to reduce these masses make them easily confused with incarcerated hernias. When encountered, they should be completely removed if possible to prevent continued hyperplasia and promote relief of discomfort.
\end{abstract}

\section{Introduction}

Inguinal cord cysts are a rare finding, comprising less than $1 \%$ of inguinal hernia dissections [1]. These are benign masses of atypical tissue found within the inguinal cord that are anatomically different from hydroceles and hernias. They deserve attention not only as an unusual finding, but for their typical presentation as incarcerated inguinal hernias and the topics of preoperative imaging and planning that they bring to the forefront. We present an atypical case and a review of the literature.

\section{Case Report}

A nineteen-year-old male with a history of a right inguinal hernia repair at the age of four, presented with a left groin lump of one-month duration that subjectively felt similar to his prior right hernia. The patient had pain with exertion, but no obstructive symptoms. He reported to his primary care provider with some erythema overlying his non-reducible bulge and he was sent to the hospital. In the emergency department, an ultrasound was performed and a non-reducible collection with heterogeneous intraluminal contents and a hernia neck was reported; consistent with a bowel-contianinghernia (Figure 1). He was consented for an inguinal hernia repair and taken to the operating room. Intraoperatively, he was found to have no hernia, but a cystic mass on the spermatic cord. The mass was intimately attached to vessels within the cord. It was dissected off the cord leaving all vessels and structures intact with a small button of tissue remaining. Final histopathological diagnosis was pseudocyst, which has not been reported in the literature (Figure 1). The patient recovered well with resolution of pain at postoperative follow up.
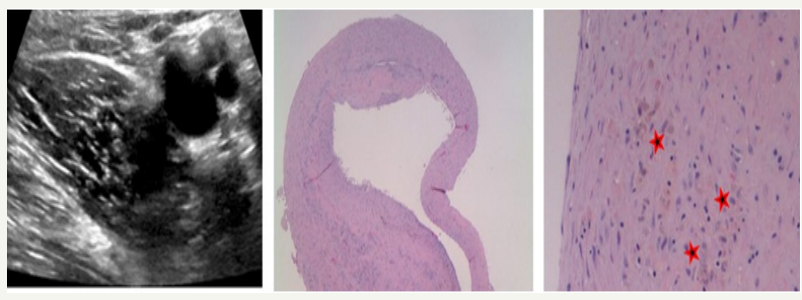

Figure 1: Ultrasound image (left) of the groin bulge demonstrates a heterogenous fluid collection in close proximity to the cord vessels. Microscopic pathology under low power (middle) showing the pseudocyst cavity on cross section. A lumen is visualized with a uniform wall of at least $1 \mathrm{~mm}$ circumferentially. High power (right) of the cyst wall does not identify a specific cell lining; it demonstrates mild acute and chronic inflammation, with hemosiderin-laden macrophages labeled by the stars.

\section{Discussion}

Inguinal cord cysts are anatomically distinct from cord lipomas and hydroceles. They are most commonly benign masses of atypical tissue. Most cysts are caused by mesothelial hyperplasia when flawed obliteration of an embryonal structure such as the process us vaginalis or canal of nuck occurs [1,2]. They are often found at the end of an indirect inguinal hernia sack. Islands of remnant embryologic tissue such as adrenal cortical rests are 
found in approximately $4 \%$ of male children undergoing surgery for inguinal hernia or undescended testes [1]. When approaching a groin mass that presents similar to an incarcerated inguinal hernia, one must have a wide differential diagnosis. Aberrant tissue has been discovered in the inguinal canal with or without hernia, these include: adrenal cortical tissue, uterine fibroids, endometriosis, ovary, pancreatic pseudocyst, lymph nodes [3]. Infectious etiology is another possibility for groin bulge and includes diverticular abscesses [3]. Traumatic etiology includes a delayed presentation of a ruptured spleen and spermatic cord hematomas [4]. Neoplasia is an etiology of high concern with the possibility of metastatic disease and includes: dermoid cysts and liposarcoma [1].

The variety of mimics of a bowel-containing incarcerated hernia makes counselling the patient important and brings up the topic of pre-operative imaging. The hernia sac is difficult to discern on ultrasound [5]. When analyzing the contents of a hernia sac omental fat is typically hyperechoic, fluid is anechoic, and bowl contents are seen as a mixed pattern due to the heterogeneous mixture of air and semisolids [5]. The ultrasound appearance for our patient is most definitely mixed and therefore may have caused confusion (Figure 1). Groin masses can also be confusing in female patients. Round ligament varicosities have been mistaken for inguinal hernias in pregnancy, resulting in unnecessary surgery in a high-risk patient [6]. This further emphasizes the need for accurate interpretation of ultrasonography.

When anon-reducible groin bulge presents it is important to keep a wide differential and analyze the clinical picture, paying close attention to the chronicity of lesions. When ordering imaging it may be beneficial to ask the radiologist to evaluate for more than just a hernia and rather a groin mass, keeping the door open for alternative diagnoses. Uncertainty can always be followed with further imaging such as CT to better prepare the surgeon and the patient for operative expectations such as a cancer operation and potential need for orchiectomy. When performing an operation on an incarcerated inguinal hernia an anterior open approach offers the best exposure. Laparoscopic and preperitoneal approaches may miss these masses as they are typically found at the distal tip of the indirect sack external to the transversalis fascia. Retained inguinal cord cysts have a high risk of continuing to cause symptoms postoperatively, resulting in reoperation. We did leave a small button of tissue yet recurrence was not seen six months postoperatively. If preoperative imaging is correctly interpreted, a difficult dissection with possible damage to testicular blood supply can be anticipated. Depending on the type of cyst found, complete excision may be required to prevent recurrence and a hernia repair may or may not be required.

\section{References}

1. Welch B, Barton T (2002) Inguinal cord cysts. Hernia 6(1): 33-35.

2. Nistal M, Iniguez L, Paniagua R (1987) Histological classification of spermatic cord cysts in relation to their histogenesis. Eur Urol 13(5): 327-330.

3. Bunting D, Harshen R, Ravichandra M, Ridings P (2006) Unusual diagnoses presenting as incarcerated inguinal hernia: A case report and review of the literature. Int J Clin Pr 60(12): 1681-1682.

4. Sherman HF (1989) The inguinal hernia, not always straightforward, not always a hernia. J Emerg Med 7(1): 21-24.

5. Park HR, Park S Bin, Lee ES, Park HJ (2016) Sonographic evaluation of inguinal lesions. J Clin Imaging 40(5): 949-955.

6. Mine Y, Eguchi S, Enjouji A, Fukuda M, Yamaguchi J, et al. (2007) Round ligament varicosities diagnosed as inguinal hernia during pregnancy: A case report and series from two regional hospitals in Japan. Int J Surg Case Rep 36: 122-125.
Creative Commons Attribution 4.0 International License

For possible submissions Click Here
Submit Article

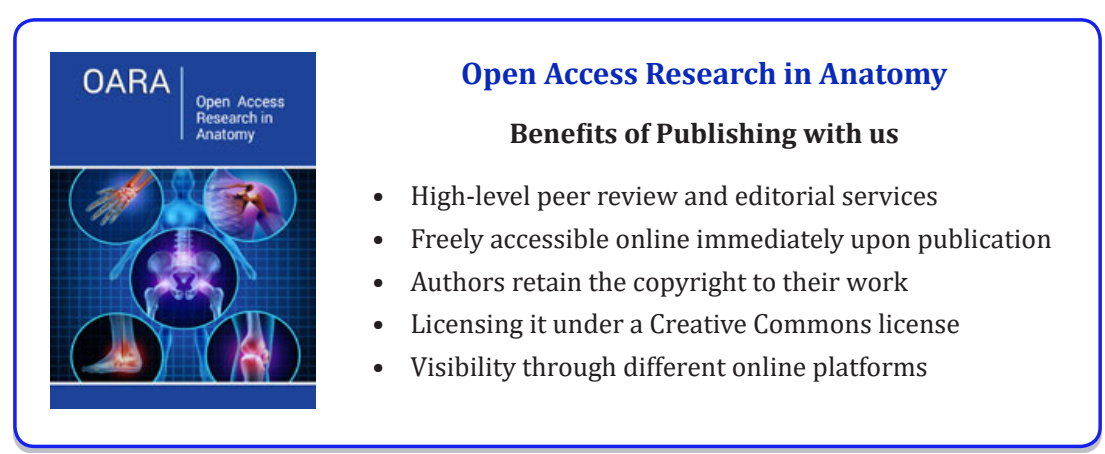

